\title{
A RARE UNILATERAL WIDESPREAD MULTIPLE FORMS OF ORAL LICHEN PLANUS: CLINICO-HISTOPATHOLOGIC EVALUATION AND DIAGNOSTIC CONSIDERATIONS: A CASE REPORT
}

\author{
Abdurahman Musbah Elmezwghi * , Abeer Hussen Elsagali ** , Salma S. Mo Zariba*** \\ Intesar Hassan Hussein Rih ${ }^{* * * *}$ and Raga Abuatwirat ${ }^{* * * * *}$
}

\begin{abstract}
Lichen planus (LP) is a chronic disease of the skin and mucous membrane. Oral lichen planus (OLP), the mucosal counterpart of cutaneous lichen planus that presents with a variety of clinical features, about $25 \%$ present with oral lesions alone. Patients may develop white striations, white papules, white plaques, erythema, erosions, or blisters affecting predominantly the posterior buccal mucosa bilaterally. The clinical presentation of OLP ranges from mild painless white keratotic lesions to painful erosions and ulcerations. The diagnosis of OLP was based on history, clinical findings and histopathological features. There is no consensus on a single set of criteria for the diagnosis of OLP. Some investigators use only the clinical criteria, while others use both clinical and histopathologic criteria. Furthermore final diagnosis was confirmed by Immunohistochemical staining (IHC) with CD8 precursor cells. The possibility of this lesion to turn malignant justifies the importance of early definitive diagnosis and long term follow up for patients with such disease.
\end{abstract}

\section{INTRODUCTION}

Oral lichen planus (OLP) is a chronic mucocutaneous disease of uncertain etiopathogenesis. $^{(1)}$ OLP usually affects $1-2 \%$ of the adult population. ${ }^{(2)}$ OLP shows a predominance among females between their fifth and sixth decade of life, ${ }^{(3)}$ although younger adults and children may be affected.(4) Lesion manifestations can often persist for years alternating between periods of quiescence and exacerbation. ${ }^{(5)}$ Exacerbations of OLP are associated with periods of psychological stress, anxiety and mechanical trauma (Koebner phenomenon). ${ }^{(6)}$ Although the etiology and pathogenesis of OLP is not well determined, genetic predisposition, stresses, as well as some of viral and bacterial agents are various factors that may act as risk factors for this disease. ${ }^{(7)}$ There is consensus that in OLP there is chronic, cell-mediated, immune

\footnotetext{
* Lecturer in Oral Pathology Department.

** Assisstant Prof. in Oral \& Maxillofacial Surgery, Department

*** Lecturer In Oral \& Maxillofacial Surgery Department

**** Lecturer In Oral \& Maxillofacial Surgery Department

***** Assisstant Lecturer In Oral \& Maxillofacial Surgery Department Faculty Of Dentistry / University Of Tripoli
} 
damage to basal keratinocytes in the oral mucosa that are recognized as being antigenically foreign or altered ${ }^{(8)}$ OLP is a T-cell mediated autoimmune disease in which the auto cytotoxic CD8+ T-cells trigger apoptosis of the basal cells of the oral epithelium. ${ }^{(9)}$ T-cells and macrophages comprise the dense sub-epithelial mononuclear infiltrate in OLP, and also there is an accentuation of T-cells within the epithelium. ${ }^{(9)}$ Recently, there is increased intentness toward the role of mast cells (MCs) in the pathogenesis of OLP. ${ }^{(10)}$ OLP may occur isolated or in combination with involvement of cutaneous sites or other mucosal sites, ${ }^{(11)}$ approximately one third of the patients who have oral lesions also have skin lesions. ${ }^{(12)}$ However, some studies report that OLP, without cutaneous involvement occurs more commonly. ${ }^{(13)}$ Intraoraly, buccal mucosa, tongue and gingiva are most commonly involved. ${ }^{(14)}$ They are usually symmetrical and bilateral lesions or multiple lesions in the mouth, ${ }^{(15)}$ while other areas like mucosa of the palate and floor of the mouth are rarely affected (16). Mucosal lesions are classified into six clinical forms, described by Andreason: Reticular, papular, plaque (white forms), the reticular form occurs more frequently and is characterized by white lacy streaks known as Wickham's striae, which generally are surrounded by discrete erythematous borders. ${ }^{(17)}$ Atrophic, erosive and bullous (red forms). ${ }^{(18)}$ The most common subtype, is usually asymptomatic.(19) Furthermore, the erosive and atrophic forms cause discomfort and painful symptoms. ${ }^{(20)}$ The papular form of OLP presents as small white pinpoint papules about $0.5 \mathrm{~mm}$ in site. It is rarely seen and being small, it is possible to overlook them during a routine oral examination. ${ }^{(21)}$ the papular type usually coexists with another type. ${ }^{(22-19)}$ The plaque form of oral lichen planus is characterized by plaque like lesions, most frequently on the dorsum of the tongue and gingiva, clinically resemble oral leukoplakia. ${ }^{(23)}$ Plaque-type affections were seen with a significantly higher frequency among tobacco smokers at the onset of OLP. (24)
Lichen planus can be diagnosed clinically in classic cases, ${ }^{(25)}$ but biopsy is recommended to confirm the diagnosis and to exclude dysplasia and malignancy. ${ }^{(26)}$ Classic histopathologic features include the presence of a lymphocytic infiltrate in the subepithelial region in band-like patterns, liquefactive degeneration of the basal layer, Civatte's bodies, variable degrees of focal ortho or parakeratosis and irregular acanthosis ${ }^{(27)}$.Several studies report the malignant potential of OLP. However, this topic is still quite controversial. ${ }^{(28)}$ The frequency of malignant transformation varies between 0 and $3.5 \%$, erythematous and erosive lesions show the highest index. ${ }^{(29)}$ Therefore every patient diagnosed with OLP should be regularly monitored since malignant transformation can occur in all forms of OLP. ${ }^{(30)}$

\section{Case Report}

A 60-years-old Libyan male patient was attended to Oral medicine clinic at Faculty of Dentistry - University of Tripoli with chief complaint of a burning sensation in the right side of his mouth since six months. Patient did not give any history of tobacco consumption or alcohol drinking. His medical and family histories were unremarkable. Also, the patient did not provide any drug history during the last 12 months, and he did not go through any dental procedure at that time. On general examination of the patient, no cutaneous lesions were evident on any other part of the body other than intraoral areas. There were no palpable lymph nodes of the head and neck regions. Routine hematological investigations and biochemistry yielded negative results. Intraoral examination revealed widespread multiple unilateral grayish- white non scrapable patches extending from the right commissure of the mouth, buccal mucosa, and lower right posterior gingival segment up to the retromolar area (fig.1a), on the right lateral border of the tongue posteriorly extending to the floor of the mouth (fig.1b). The left buccal mucosa as well as other sites of the oral 
cavity appeared normal (fig.2). Lesions represented by unilateral irregular white reticular with striations and white plaque forms (fig.3 a and b). The symptoms of discomfort were exacerbated by spicy foods. The differential diagnosis can include cheek chewing/frictional keratosis, lichenoid reactions, leukoplakia, lupus erythematous, pemphigus, mucus membrane pemphigoid, para neoplastic pemphigus, erythematous candidiasis and chronic ulcerative stomatitis, Graft vs. host disease.

However, In order to lead to a final diagnosis an incisional biopsy specimens were subjected to routine hematoxylin and eosin $(\mathrm{H} \& \mathrm{E})$, and immuno-histochemical staining (IHC) staining was performed by using CD8+Tcell to confirm the final diagnosis.

Three pieces of soft tissue specimen was received measuring about $1.0 / 0.8 / 0.5 \mathrm{~cm}$ in size. They were roughly oval in shape grayish white in color. The tissues were soft in consistency with smooth surface and regular border.

Histopathological examination with $\mathrm{H} \& \mathrm{E}$ stain revealed parakeratinized stratified squamous epithelium along with the underlying connective tissue stroma. The epithelium was atrophic showing absence of rete pegs. Focal areas of the epithelium also showed degeneration of basal cells. A band of juxta epithelial inflammatory cell infiltrate was evident, chiefly comprising of lymphocytes and plasma cells (fig.4). IHC staining showed subepithelial immunopositivity reaction for CD8 + T cells (fig.5). Based on the histopathologic findings, were consistent with the diagnosis of unilateral oral lichen planus. The patient was advised to avoid eating spicy foods while his lichen planus was active and to use topical corticosteroid cream containing 0.1 percent triamcinolone acetonide (Kenacort), when symptomatic. Initial review after a threemonth period showed a reduction in symptoms and severity of the reticular lichen planus. The patient is currently continuing follow-up.

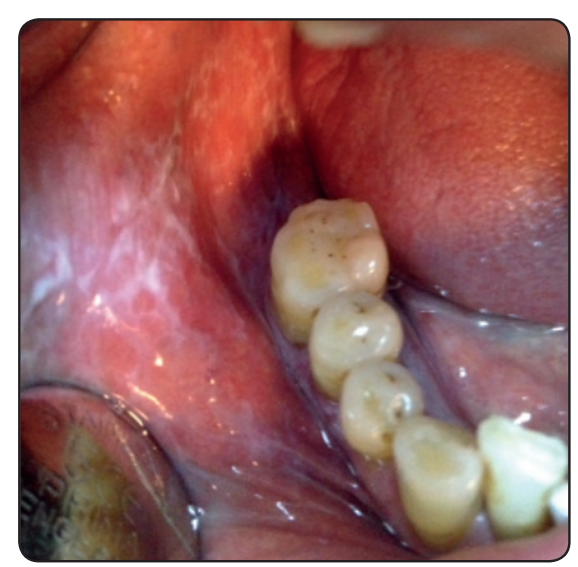

Fig. (1a) Widespread multiple unilateral grayish- white non scrapable patches extending from the right commissure of the mouth, buccal mucosa, lower right posterior buccal vestibule, posterior gingival segment up to the retromolar area.

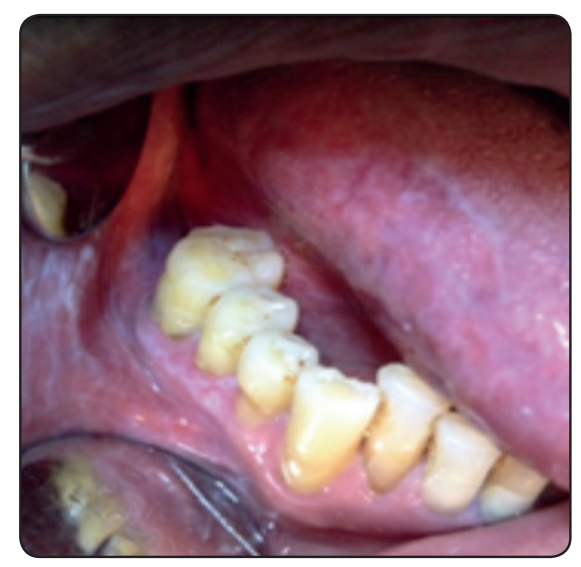

Fig. (1b) Widespread multiple unilateral grayish- white non scrapable patches on the right lateral border of the tongue posteriorly extending to the floor of the mouth

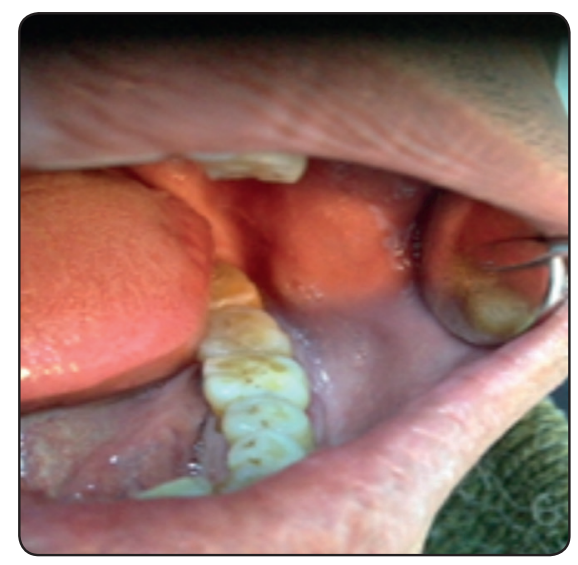

Fig. (2) The left buccal mucosa as well as other sites of the oral cavity appeared normal 


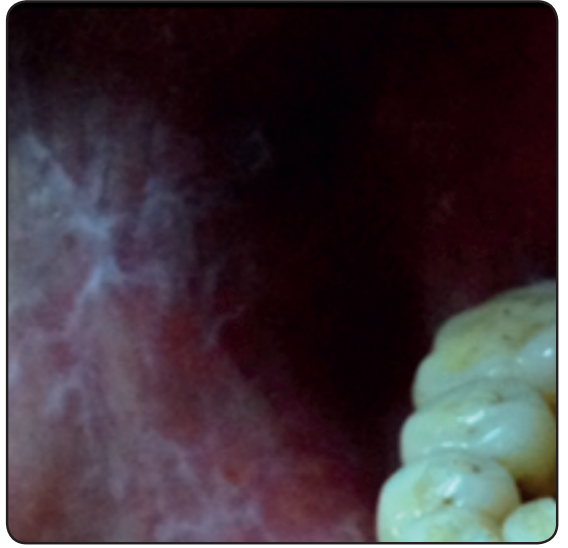

Fig. (3a) Reticular pattern showing white lines of striae on the buccal mucosa.

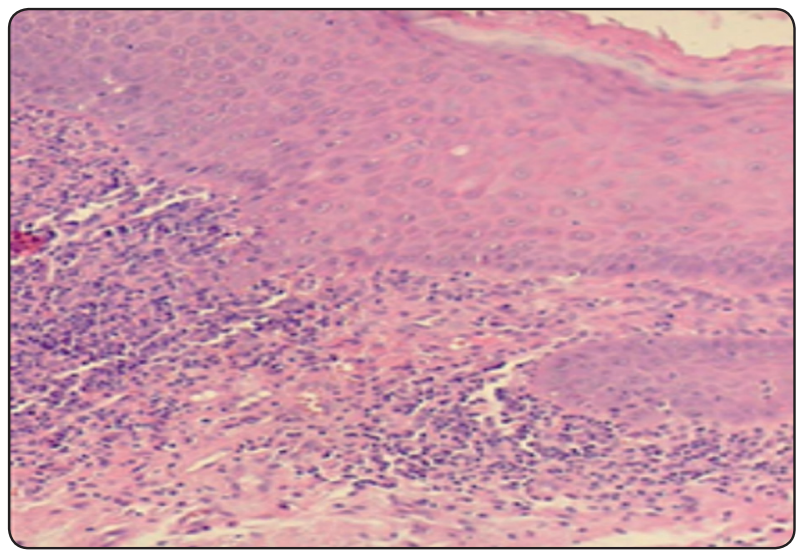

Fig. (4) A photomicrograph showing parakeratinized squamous epithelium, the epithelium is atrophic with absence of rete pegs, a band of subepithelial Inflammatory cell infiltrate and focal area of basal cells degeneration (H\&E X20).

\section{DISCUSSION}

LP was first described by Wilson in $1869 .{ }^{(31)}$ In 1895, Thieberg identified the oral lesion. ${ }^{(32)}$ OLP is a common chronic immunological, inflammatory mucocutaneous disorder. ${ }^{(33)}$ Although the etiology and pathogenesis of OLP is not well-determined, genetic predisposition, stresses, as well as some of viral and bacterial agents are various factors that may act as risk factors for this disease. ${ }^{(7)}$ In the presented case our patient was not taking any medication, the patient did not go through any dental treatment, he did not give any history of tobacco

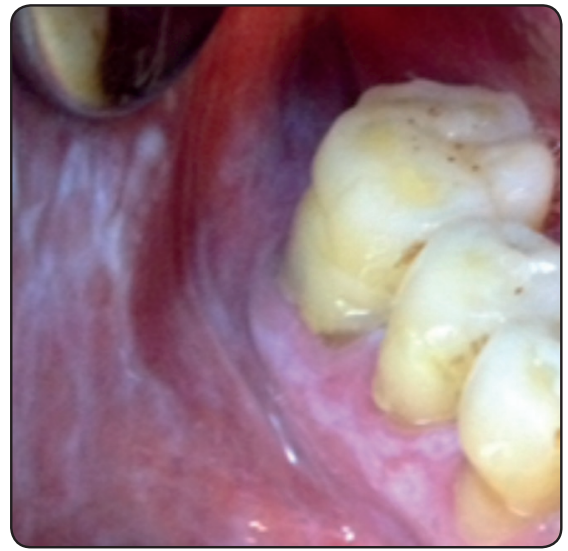

Fig. (3b) Plaque pattern showing a homogenous white plaque in conjunction with striae on buccal mucosa and gingiva

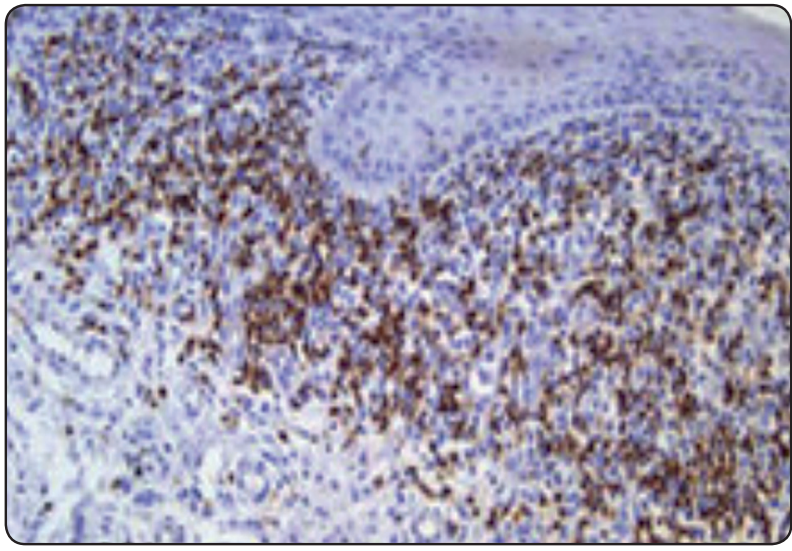

Fig. (5) A photomicrograph of reticular oral lichen planus showing subepithelial immunopositivity reaction for CD8 +Tcell. (Immunohistochemical, X20)

consumption or alcohol drinking, and his medical and family histories were unremarkable, so the cause-effect relationship could not be established. About half of the patients with skin lesions have oral lesions, whereas about $25 \%$ present with oral lesions alone. ${ }^{(21)}$ (OLP) is reported to occur more frequently than the cutaneous form ${ }^{(34)}$. In contrast to cutaneous lichen planus, the oral form may persist for up to 25 years. $^{(15)}$ In our case no cutaneous lesions were evident on any other part of the body other than intraoral areas, this finding was coincide with many previous studies. The typical age of 
presentation is between 30 and 60 years, and it is more frequently seen in women. ${ }^{(35)}$ The prevalence of OLP in children is rare. ${ }^{(18)}$ The age of our patient in this case report was 60 years, which fall within age range in which lichen planus is most commonly reported. The reticular type has been reported to occur significantly more often in men compared to women. ${ }^{(36)}$ Our patient in this presented case was male with reticular oral lichen planus is in agreement with Chainani-Wu N., et al (2001), ${ }^{(37)}$ who studied 229 cases of OLP. The mucosa of the palate and floor of the mouth is rarely affected. ${ }^{(38)}$ In our report case the lesion was extended to involve the floor of the mouth on the right side only, this rare finding have been reported in previous studies. The reticular form is the most common type and presents as papules and plaques with interlacing white keratotic lines (Wickham's striae) with an erythematous border. The striae are typically located bilaterally and is usually asymptomatic on the buccal mucosa, mucobuccal fold, gingiva, and less commonly, the tongue, and lips. ${ }^{(36)}$ The erythematous forms are painful, causing discomfort to the patient ${ }^{(39)}$. The second most common pattern was the plaque pattern. ${ }^{(28)}$ Plaque forms appear as homogenous white patches resembling leukoplakia, ${ }^{(40)}$ often but not always surrounded by striae. ${ }^{(41)}$ Plaque-type was a more constant form, but also demonstrated many newly established affections, ${ }^{(24)}$ it mainly involves the dorsum of the tongue and the mucosa of the cheek. ${ }^{(42)}$ In our presented cases the anatomical sites of the lesion were unilateral, involved commissure of the mouth, buccal mucosa, buccal vestibule, retromolar pad, lateral border of the tongue, and floor of the mouth, had a striae shape and were reticulated whitish, the gingival lesion had a whitish and purple plaques. Our case can be considered rare and unique according to its widespread unilateral occurrence, reticular and plaque pattern showed symptomatic lesion and involvement of the floor of the mouth, which has been reported to be less common. In the classic lesions, it is possible to make a diagnosis based on its clinical appearance alone ${ }^{(\mathbf{1 0})}$. In the absence of typical manifestations of the reticular pattern and other patterns may be difficult to diagnose. ${ }^{(43)}$ However, biopsy is recommended to confirm the diagnosis and to exclude dysplasia and malignancy, ${ }^{(26)}$ which was performed in our case.

Histopathological features of evident OLP are a varying degree of focal hyperkeratosis or parakeratosis, irregular acanthosis or atrophy, liquefaction degeneration of the basal cell layer and a dense band-like lymphocytic infiltrate high in the lamina propria. Hyaline Civatte's bodies, which represent degenerated basal cells, are occasionally seen in the epithelium ${ }^{(44)}$. Histopathological features were observed in this presented case study were consistent with the diagnosis of typical OLP and similar to other reports. Lymphocytic infiltration and keratinocyte apoptosis has been observed to likely promote the activation of a cell-mediated immune response ${ }^{(45)}$. The infiltration of OLP is mainly represented by CD8 + suppressor-cytotoxic cells. Previous studies have showed that CD $8+\mathrm{T}$ cells constitute a high proportion of the cellular infiltrate in OLP. ${ }^{(46)}$ In this presented case report, IHC CD8 Tcell marker was performed to confirm the definite diagnosis, and exclude any dysplastic changes and showed subepithelial immunopositivity reaction.

According to the clinico-histoplathological assessment of our presented case, the differential diagnosis can include lichenoid reaction (LR) (but our patient did not provide any drug history during the last 12 months nor go through any dental procedure at that time. Clinically, LR has tendency to be localized and asymmetrically distributed. Furthermore, biopsy suggestive of diffuse lymphocytic infiltrate rather than a subepithelial band which was evident in our case). Leukoplakia (due to thick whitish plaque appearance of the lesion in the attached gingiva, but excluded on the basis of typical histopathological features of the presented lesion). Cheek chewing/frictional keratosis (but the 
lesion was widespread extended beyond the occlusal line and no sharp tooth or dental appliance related to the site of the lesion). lupus erythematosus (LE) (Clinically, lesions of LE most often resemble erosive lichen planus, but our case was of typical reticular and plaque clinical features, The keratotic striae of LE are much more delicate and subtle than Wickham's striae and show a characteristic radiation from the central focus rather than peripheral radiation which was exhibited clearly in our presented case. Furthermore, histopathologic examination did not show characteristic of perivascular infiltrate. World Health Organization (WHO) classified OLP as a potentially malignant disorder. ${ }^{(47)}$ The malignant transformation occurs most frequently in the erosive form of OLP (35\%), followed by the plaque type (24\%) and the reticular type $(11 \%) .{ }^{(48)}$ Our patient did not smoke nor drink alcohol without family history of any type of cancer. The malignant transformation of OLP cannot be predicted. Therefore, it is essential to keeping the patients on close and precise follow up.

\section{RECOMMENDATION}

The typical clinical features of oral lichen planus are usually sufficient for the diagnosis of this condition. Still, a biopsy for histopathology is recommended to confirm the clinical diagnosis and mainly to exclude epithelial atypia and signs of malignancy. In the absence of typical manifestations of the reticular pattern, other patterns may be difficult to diagnose. In such cases, a cytopathology proved to be an important tool for diagnosis. When the histopathologic features are suspicious, Immunohistochemical staining by using CD4 and CD8 markers are recommended as one of the precise diagnostic tools to confirm the final diagnosis. In case of changes in the appearance of OLP lesion, follow-up, further biopsies should be carried out, due to their malignant potential. We recommended a follow up of at least one or two visits per year as long as OLP persists.

\section{REFERENCES}

1. Simrata Ajrawat.et al. Erythematous Oral Lichen Planus in an Ongoing Orthodontic Treatment. Univ Res J Dent 2016;6:72-75.

2. Teja CR.et al. Cytomorphometric analysis of exfoliated cells in oral lichen planus. CytoJournal 2014;11:3-10.

3. Oliveira Alves. et al. Oral lichen planus: A retrospective study of 110 Brazilian patients BMC Research Notes. 2010;3:157-60.

4. PB Sugerman, and NW Savage. Oral lichen planus: Causes, diagnosis and management. Australian Dental Journal. 2002;47( 4):290-297.

5. Wang, K. et al. Preliminary analysis of salivary microbiome and their potential roles in oral lichen planus. Sci. Rep. 2016; 6:1-10.

6. Canto AM, Muller H, Freitas RR, da Silva Santos PS. Oral lichen planus (OLP): clinical and complementary diagnosis. An Bras Dermatol. 2010;85(5):669-75.

7. Amirchaghmaghi M. et al. Evaluation of Plasma Isoprostane in Patients with Oral Lichen Planus. J Dent Shiraz Univ Med Sci.2016;17(1):21-25.

8. Thornhill MH. Immune mechanisms in oral lichen planus. Acta Odontol Scand J. 2001 Jun;59(3):174-7.

9. Bibin Thomas George. et al. Oral Lichen Planus: A Case Report and an Update on the Role of Mast Cells in its Pathogenesis. J Inte Oral Health.2016; 8(2):292-295.

10. Fernando Augusto Cervantes Garcia de Sousa, Luiz Eduardo Blumer Rosa. Oral lichen planus: clinical and histopathological considerations.Rev Bras J Otor.2008; 74(2):284-92.

11. Jing Wang, Isaäc van der Waal. Disease scoring systems for oral lichen planus; a critical appraisal. Med Oral Patol Oral Cir Bucal.2015;20(2):199-204.

12. Diana Mostafaa, and Bassel Tarakjia. Photodynamic Therapy in Treatment of Oral Lichen Planus. J Clin Med Res.2015;7(6):393-399.

13. Chatterjee K, Bhattacharya S, Mukherjee CG, Mazumdar A. A retrospective study of oral lichen planus in paediatric population. J Oral Maxillofac Pathol. 2012; 16:363-7.

14. Salvador Arias-Santiago, et al. Cardiovascular Risk Factors in Patients with Lichen Planus.The American Journal of Medicine.2011;124:543-548.

15. Manas Bajpai. ert al. Unilateral lichen planus: A rare case report. J Nat Sci Biol Med. 2014;5(2):453-455.

16. Oliveira Alves MG, Almeida JD, Balducci I, Guimarães 
Cabral LA. Oral lichen planus: A retrospective study of 110 Brazilian patients. BMC Res Notes.2010;3:157.

17. Edwards PC, and Kelsch R. Oral lichen planus: Clinical presentation and management.J Can Dent Assoc 2002;68(8):494-9.

18. Jayanna R.et al.Clinical Features, Diagnosis and Management of Oral Lichen Planus in Children. J Indian Aca Oral Med Radiol.2013;25(3):209-213.

19. Farzam Gorouhi. et al. Cutaneous and Mucosal Lichen Planus:A Comprehensive Review of Clinical Subtypes, Risk Factors, Diagnosis, and Prognosis. The Scientific World Journal. 2014, Article ID 742826, 22 pages.

20. Santosh Patil. et al.Treatment modalities of oral lichen planus: an update. J. Oral Diag. 2012;1(2):47-52.

21. Rajendran R. Oral lichen planus. J Oral Maxillofac Pathol. 2005;9:3-5.

22. F. A. de Sousa and L. E. B. Rosa.Oral Lichen Planus: clinical and histopathological considerations. Braz J Otor. 2008;74(2):284-292.

23. Sanja Persic. et al. Oral Lesions in Patients with Lichen Planus. Acta Clin Croat. 2008;47:91-96.

24. Thorn JJ. et al. Course of various clinical forms of oral lichen planus. A prospective follow-up study of 611patients.J Oral Pathol.1988;17(5): 213-8.

25. Richard P.Usatine, and Michelle Tinitigan. Diagnosis and Treatment of. Lichen Planus. Am Fam Physician. 2011;84(1):53-60.

26. Scully C, and Carrozzo M. Oral mucosal disease: Lichen planus. Br J Oral Maxillofac Surg. 2008;46(1):15-21.

27. Dr. Gopal Sharma. et al. A Rare Case Report of Erosive Lichen Planus of Tongue Treated with Lycopene. Inte J Curr Res.20013;5(10):3192-3194.

28. Werneck JT.et al Oral lichen planus:study of 21 cases. An Bras Dermatol. 2015;90(3):321-6.

29. Fitzpatrick SG, Hirsch SA, and Gordon SC.The malignant transformation of oral lichen planus and oral lichenoid lesions: a systematic review. J Am Dent Assoc.2014; $145: 45-56$.

30. Kaplan I.et al. The dynamics of oral lichen planus: a retrospective clinicopathological study. Head Neck Pathol. 2012;6:178-83.

31. Paul M, Shetty DC. Analysis of the changes in the basal cell region of oral lichen planus:An ultrastructural study.J Oral Maxillofac Pathol 2013;17:10-6.

32. Archana Patil, Shiva Prasad, L. Ashok, and G. P. Sujatha.
Oral bullous lichen planus: Case report and review of management. Contemp Clin Dent. 2012;3(3):344-348.

33. Greenberg MS, Glick M, Ship JA. Red and white lesions of oral mucosa. Burkets Oral Medicine.12th ed. PMPHUSA, LTD; 2015.

34. Doulat Rai Bajaj.et al.Oral Lichen Planus: A Clinical Study. J Col Phys and Surg Paki. 2010;20(3):154-157.

35. Ibtisam Al-Hashimi. et al. Oral lichen planus and oral lichenoid lesions: diagnostic and therapeutic considerations. Oral Surg Oral Med Oral Pathol Oral Radiol Endod. 2007; 103(1): S25. e1-S25. e12.

36. M GunaShekhar.et al. Oral lichen planus in childhood: A rare case report. Derma Online J.2010;16(8):9.

37. Chainani-Wu N. et al. Oral lichen planus: patient profile, disease progression and treatment responses.J Am Dent Assoc.2001;132(7):901-909.

38. Ingafou M, Leao JC, Porter SR, and Scully C: Oral lichen planus: a retrospective study of 690 British patients. Oral Dis .2006;2:463-8.

39. Maiara de Moraes. et al. Oral lichen planus: two case reports in male patients. Rev. odonto ciênc. 2010; 25(2):208-212.

40. Kanemitsu Shirasuna. Oral lichen planus: Malignant potential and diagnosis. Oral Science International J. 2014;11(1):1-7.

41. DeRossi SS, and Ciarrocca KN. Lichen planus, lichenoid drug reactions, and lichenoid mucositis. Dent Clin North Am.2005;49:77-89.

42. Gupta S, Jawanda MK. Oral lichen planus: An update on etiology, pathogenesis, clinical presentation, diagnosis and management. Indian J Dermatol 2015;60:222-9.

43. van der Waal I. Oral lichen planus and oral lichenoid lesions; a critical appraisal with emphasis on the diagnostic aspects. Med Oral Patol Oral Cir Bucal.2009;14:E310-4.

44. Ronald Laeijendecker. et al. Premalignant Nature of Oral Lichen Planus. Acta Derm Venereol. 2005;85:516-520.

45. Sedigheh Bakhtiari. et al. Assessment of Uric Acid Level in the Saliva of Patients with Oral Lichen Planus.Med Princ Pract 2017;26:57-60.

46. N Lavanya. et al. Oral lichen planus: An update on pathogenesis and treatment. J Oral Maxillofac Pathol.2011; 15(2):127-132.

47. Soma Susan Varghese. et al. Epidemiology of Oral Lichen Planus in a Cohort of South Indian Population: A Retrospective Study. J Cancer Prev. 2016;21(1):55-59.

48. S. Irani. Squamous Cell Carcinoma arising in Oral Lichen Planus. DJH. 2010;1(2):49-52. 